Relations industrielles

Industrial Relations

\title{
Long, Bonita C., and Sharon E. Kahn, editors, Women, Work, and Coping
}

\section{Érick Chamberland}

Volume 50, numéro 1, 1995

URI : https://id.erudit.org/iderudit/051007ar

DOI : https://doi.org/10.7202/051007ar

Aller au sommaire du numéro

Éditeur(s)

Département des relations industrielles de l'Université Laval

ISSN

0034-379X (imprimé)

1703-8138 (numérique)

Découvrir la revue

Citer ce compte rendu

Chamberland, É. (1995). Compte rendu de [Long, Bonita C., and Sharon E. Kahn, editors, Women, Work, and Coping]. Relations industrielles / Industrial

Relations, 50(1), 239-242. https://doi.org/10.7202/051007ar

Tous droits réservés @ Département des relations industrielles de l'Université Laval, 1995
Ce document est protégé par la loi sur le droit d'auteur. L’utilisation des services d'Érudit (y compris la reproduction) est assujettie à sa politique d'utilisation que vous pouvez consulter en ligne.

https://apropos.erudit.org/fr/usagers/politique-dutilisation/ 
fort compréhensible. Mais, il devrait peut être en être autrement lorsqu'on offre au public semblable ouvrage sous un titre quasi générique: "Le droit disciplinaire des corporations professionnelles "!

Le chapitre préliminaire traite du caractère autonome du droit disciplinaire (p. 9 à 34). L'auteur déclare qu'il y élabore une "théorie de l'autonomie du droit disciplinaire" (p. 9). Au-delà des sous-titres et de quelques assertions, nous n'y avons guère trouvé pareille élaboration, mais seulement une courte démonstration du particularisme de ce volet spécifique du droit. Distinct du droit pénal, exclu du droit privé, ce droit disciplinaire est certes une composante du droit administratif et soumis au respect des chartes des droits et libertés. Il est, en quelque sorte, l'armature juridique au soutien de la déontologie des professionnels. Compte tenu de sa spécificité, ce processus de contrôle par ses pairs n'écarte pas l'application parallèle de la justice pénale ou civile: "Un professionnel peut être poursuivi pour le même acte, ou la même omission, à la fois au pénal, au civil et devant une instance disciplinaire, car une faute disciplinaire ne constitue pas nécessairement une infraction pénale et n'entraîne pas nécessairement une responsabilité civile. L'inverse est également vrai " ( $p$. 24).

La première partie de l'ouvrage ( $p$. 35 à 82) porte sur la faute disciplinaire que l'on définit comme un "manquement volontaire ou d'une certaine gravité à un devoir professionnel " (p. 39). À notre avis, le caractère expressément volontaire d'un acte fautif professionnel aggrave certes l'acte reprochê mais celuici est néanmoins un manquement! On souligne cependant que le manquement reproché doit pouvoir être normalement et raisonnablement déduit du code de déontologie de la profession visée: "Le texte devrait ētre compréhensible aux yeux du professionnel et correspondre à un devoir d'ordre général, tel qu'édicté par le pouvoir habilitant " (p. 41). L'auteur explique le caractère juridique de la faute professionnelle et qu'il distingue de la faute pénale (p. 46). Ce manquement professionnel est rattaché à la personne même de l'auteur sans égard au lieu où il est réalisé, ni au contexte collectif qui l'explique (exemple : la grève) (p. 48) et ni même au fait qu'il entraîne ou non des dommages à d'autres personnes (p. 49). Le chapitre II (p. 61 à 82 ), serait une classification des fautes disciplinaires. Sous ce titre, on y retrouve davantage une synthèse de la jurisprudence de différents comités de discipline et du Tribunal des professions. Ces manquements sont sériés en trois catégories : compétence, intégrité et dignité.

La deuxième partie du livre (p. 83 à 115) porte sur la sanction disciplinaire. L'auteur démontre que l'approche alors retenue par les comités de discipline et le Tribunal des professions serait de nature pénale et regrette une telle conception qui occulte l'effet correctif de la sanction: "Le but du contrôle disciplinaire n'est pas la punition mais bien la protection du public " (p. 84). Dans sa conclusion, l'auteur reprend le thème de l'autonomie du droit disciplinaire sans davantage justifier ni expliciter le sens et la portée de cette autonomie.

FERNAND MORIN Université Laval

\section{Women, Work, and Coping}

edited by Bonita C. LONG and Sharon E. KAHN, Toronto, McGill-Queen's

University Press, 1993, 332 p., ISBN 0-7735-1128-8 (cloth),

ISBN 0-7735-1129-6 (paper).

Parmi les femmes qui sont confrontées au stress dans leur emploi, certai- nes en retirent une plus grande satisfaction que d'autres qui ne travaillent pas 
hors de la maison. Cela implique que les femmes qui travaillent ont réussi à développer des ressources efficaces et des stratégies d'ajustement pour contrer ces formes de stress.

Cet ouvrage collectif explore ce paradoxe en mettant l'emphase à la fois sur les stratégies que les femmes ont développé et sur le contexte socioculturel dans lequel les femmes qui ont un emploi rémunéré évoluent. Plusieurs thèmes ressortent des cinq sections qui regroupent quatorze chapitres. L'originalité de l'œuvre provenant de l'amalgame des contributions de la pluralité d'auteurs, il convient ici de les survoler brièvement.

Les réalités auxquelles font face les femmes en fonction de la dualité de leurs rôles professionnels et familiaux ainsi que l'hétérogénéité de leur culture, de leur âge et de leur mode de vie font l'objet de la première partie.

Le premier chapitre décrit comment la formation des femmes et leur participation au marché du travail ont changé depuis qu'elles occupent des emplois dits non traditionnels. L'auteure prédit que si les demandes grandissantes auxquelles les femmes font face dans leur double responsabilités ne sont pas atténuées par des changements dans les lieux de travail et par une plus grande participation des hommes aux tâches ménagères, ces dernières devront abandonner leur travail en dehors de la maison. Le deuxième chapitre présente les bénéfices que les femmes retirent des différents types d'emplois qu'elles occupent. Suite à une argumentation convaincante de variations culturelles de la dichotomie entre travail rémunéré et non rémunéré, les lecteurs sont invités à adopter une vision holistique du rôle des femmes et des méthodologies dites qualitatives qui soient en mesure de transcender les cultures. Le troisième chapitre offre un résumé de la littérature sur les différentes stratégies d'ajustement usuelles et non usuelles utilisées par les femmes contre le stress en milieu de travail. Ces stratégies, dites à la fois structurelles et individuelles (par exemple, le réaménagement des emplois ou les sessions de formation), sont analysées selon leurs limites respectives.

Les auteurs ont convenu que les bouleversements sociaux et culturels qui ont affecté les rôles professionnels et familiaux des femmes ont obligé ces dernières à développer de nouvelles stratégies d'ajustement au stress. Leur deuxième section élabore sur ces stratégies (coping) et les interrelations entre le contexte (structurel ou culturel de l'organisation) et l'individu. À partir d'une perspective théorique féministe, il est fait état de l'expérience des femmes dans diverses occupations, tantôt dans des structures hostiles aux femmes, tantôt dans des structures qui leurs sont favorables.

Le quatrième chapitre porte sur les différentes formes de pouvoir utilisées par les hommes et les femmes en relation avec les stratégies d'ajustement au stress employées par les femmes en milieu de travail. Selon l'auteure, les femmes ne sont toujours pas capables d'atteindre les échelons supérieurs des organisations à défaut d'avoir un véritable accès aux sphères du pouvoir. Dans la même veine, il est dit au chapitre suivant que les femmes doivent lutter contre des cultures organisationnelles patriarcales réfractaires au changement. Un modèle descriptif à quatre étapes sert à présenter les stratégies d'ajustement au stress que les femmes utilisent pour composer avec de telles cultures. Le sixième chapitre discute de l'impact du changement technologique et de la relation entre les dirigeants et leurs secrétaires sur les femmes. Cette étude illustre bien le fait que la recherche qualitative met en évidence toute l'importance du contexte d'emploi et, par conséquent, elle se démarque d'une étude quantitative traditionnelle.

Bien qu'une perspective structurelle suggère plusieurs moyens d'intervention, 
il est nécessaire de prendre en considération une perspective individuelle afin de bien saisir le phénomène du stress au travail et les différentes stratégies utilisées pour le contrer. Et le livre ni manque pas. Après avoir fait remarquer que les femmes, exposées à des formes de stress plus nombreuses et plus variées, ne semblent pas être plus affectées par le stress que leurs collègues masculins, la troisième section traite des modérateurs du stress.

Le chapitre sept offre une explication aux précédentes observations. Il y est proposé que les femmes ont, d'une part, développé des stratégies efficaces d'ajustement au stress et que, d'autre part, elles ont recours à des formes de support social. Quant au chapitre huit, on y traite des différences qui existent quant au support social offert à divers groupes de travailleurs et de travailleuses. Il est proposé que la compétence interpersonnelle soit une ressource de première importance pour les femmes. Ensuite, le contributeur du chapitre neuf démontre les bénéfices que les jeunes femmes retirent de certaines combinaisons de rôles matrimoniaux et parentaux. Enfin, la preuve est faite au chapitre dix que les perceptions des individus à l'égard du contrôle qu'ils exercent sur le stress relié à leur emploi peut diminuer ou accentuer les effets du stress.

La quatrième section présente des analyses des facteurs qui expliquent dans quelle mesure les stratégies d'ajustement sont efficaces. Les chercheurs qui ouvrent à ce niveau étudient les effets médiateurs des stratégies d'ajustement sur la relation qui existe entre le stress et ses effets. En guise d'assise théorique pour les analyses subséquentes, le chapitre onze contient une description de la théorie cognitive et relationnelle du stress de Richard Lazarus. Conformément à cette théorie, l'attention des chercheurs est portêe sur la signification personnelle des événements générateurs de stress ainsi qu'à l'étude des valeurs et des idéaux, le tout afin de mieux com- prendre quelles sont les stratégies d'ajustement qui sont les plus susceptibles d'être efficaces. En se basant sur la même théorie supposément non influencée par le sexe, l'auteur du chapitre douze ajoute à la précédente énumération les émotions et les attributions dans le processus même d'appréciation de ces stratégies d'ajustement. Pour ces chercheurs, la prochaine étape consistera à accumuler un support empirique crédible.

Finalement, la dernière section porte sur l'identification des méthodes de recherche qui sont adéquates en raison de la complexité du proçessus d'ajustement au stress. Différentes méthodes de recherche communément utilisées pour étudier le stress lié au travail et les stratégies d'ajustement sont critiquées au treizième chapitre. En plus d'y suggérer des méthodes innovatrices, les deux auteurs soulignent également les problèmes chroniques inhérents à l'étude des facteurs de stress et des stratégies d'ajustement. Afin de consolider l'ensemble des liens déjà évidents, Bonita C. Long et Sharon E. Kahn intègrent toutes les contributions en les replaçant dans le contexte du modèle de Richard Lazarus (Lazarus et Folkman, 1984). Ce faisant, elles mettent en perspective une multitude de facteurs mais surtout leurs relations qui sont d'une importance capitale dans la compréhension de la façon dont les femmes élaborent leurs stratégies d'ajustement face au stress au travail.

Force est de constater qu'il n'y a pas d'écart entre le titre et le contenu du livre. Dix-neuf auteurs de lieux de travail et d'appartenances professionnelles différents ont écrit 14 chapitres regroupés en cinq parties autour d'une péoccupation unique: l'expérience que les femmes vivent face au stress en milieu de travail et les stratégies qu'elles mettent de l'avant pour y remédier. On aurait pu craindre à un éclatement ou un éparpillement des apports, mais il n'en est rien. Les intérêts convergents des auteurs, 
l'objectif opérationnel du volume et sa structure ont su éviter la fragmentation du sujet. Les chapitres, de longueur (une vingtaine de pages), de discours et de structures passablement uniformes, présentent donc la matière de manière utile à l'initié et accessible pour le noninitié. Le volume est aussi très facile à consulter grâce aux deux index (par sujets et par auteurs).

Que retirer en définitive de cet ouvrage sinon un enseignement sur la construction d'un discours multidisciplinaire. Il faut donc lui reconnaître deux mérites: la richesse et la variété théorique des articles si bien intégrés ainsi que la finesse des analyses et critiques présentées. Ce livre constitue pour les chercheurs, les étudiants engagés et les gestionnaires une source de réflexion susceptible de les aider à mieux comprendre la façon dont les femmes gèrent le stress lié au travail.

ÉRICK CHAMBERLAND Université Laval

\section{Projecting Capitalism: A History of the Internationalization of the Construction Industry}

by Marc Linder, Westport, Connecticut, Greenwood Press, 1994, 270 p., ISBN 0-313-29293-0 and ISSN 0084-9235.

Voici un ouvrage qui aborde l'industrie de la construction d'une façon originale et fort instructive. En utilisant une approche historique, l'auteur tente de détruire le mythe que la construction est une industrie locale regroupant plusieurs petites entreprises en parfaite concurrence et à l'abri des marchés mondiaux.

Pour étayer sa thèse, l'auteur poursuit trois objectifs interreliés. D'abord, il utilise le développement d'un marché mondial pour démontrer à quel point la construction ressemble beaucoup plus aux autres industries eu égard aux impératifs structuraux, aux contraintes d'accumulation de capital et à la concentration que la vision traditionnelle le reconnaît. Ensuite, l'auteur vise à démontrer le rôle concret des entreprises de construction dans la production et l'implantation de capital fixe par les autres industries à l'extérieur des États-Unis. Finalement, l'auteur vise à présenter une perspective historique de la transformation physique et de la reconstruction du Tiers Monde dues au transfert de technologie, d'ingénierie de construction en provenance des pays industrialisés dans le contexte d'une économie mondiale de marché orientée vers l'exploitation des richesses naturelles.

Pour atteindre ses objectifs, l'auteur divise son livre en trois parties. La première présente le cadre théorique utilisé et s'attarde à l'applicabilité à la construction de la distinction, commune aux autres industries, entre les exportations et l'investissement direct à l'étranger. La seconde partie présente l'histoire des activités transnationales de construction, d'entreprises américaines et européennes avant la Seconde Guerre, remontant même jusqu'à la construction du chemin de fer britannique dans les années 1830. La troisième partie est consacrée à l'évolution d'un marché mondial pour la construction.

Linder présente ici une facette de l'industrie de la construction que nous avons trop facilement tendance à oublier. Son analyse de la préfabrication dans tous les secteurs de la construction est fort instructive. Elle a le mérite de jeter une lumière claire sur un phénomène que d'aucuns ne voient pas ou ne veulent pas voir, comme cela fut d'ailleurs le cas pour les changements technologiques dans l'industrie de la construction. 